\title{
Trial success spurs planning for rollout of HIV prevention pills
}

BOSTON - The antiretroviral pill Truvada topped Time magazine's list of medical breakthroughs last year after a study showed that taking the pill daily can prevent HIV infection. The drug, made by California's Gilead Sciences, could provide a powerful new tool to curb the worldwide AIDS epidemic. But, as HIV experts discussed here at the annual Conference on Retroviruses and Opportunistic Infections last month, questions of cost effectiveness and drug resistance, among others, still need to be answered before the product is ready for primetime.

In November of last year, an international team led by Robert Grant, a virologist at the University of California-San Francisco (UCSF), reported that the strategy, called preexposure prophylaxis (PrEP), led to a $44 \%$ reduction in HIV acquisition in a trial of nearly 2,500 sexually active gay men in six countries (N. Engl. J. Med. 363, 2587-2599, 2010). But that finding was based on data collected over only 16 months on average. "After the publication, there were questions about how durable the results would be," says Grant. So the researchers tracked the study subjects in the so-called iPrEx study for three more months, crunched the numbers and found that the efficacy of the preventative pill was still $42 \%$ on average, as Grant reported here.

The protection rate seen in the iPrEx study was much higher among people who regularly took their Truvada pills. But monitoring adherence remains a major challenge in trials of self-administered medicines. This became even clearer when Rivet Amico, a public health researcher at the University of Connecticut-Storrs, and her colleagues analyzed drug concentrations in the blood of around 180 HIV-negative study participants. The researchers found that none of the trial's indicators of pill use-including investigator-initiated counts of pills or bottles and self-reported data-fully matched direct measurements of drug concentration in the blood.

Further probing the same data set, Peter Anderson, a clinical pharmacologist at the University of Colorado-Denver, found that older people, people in the US and trial participants who engaged in the riskiest form of sex were the most likely to have detectable amounts of antiretroviral drugs in their blood, perhaps the most accurate indicator of pill use. "Using drug levels really helped us understand the iPrEx results and the need for understanding drug-taking behavior better," says Anderson.

Given the patchy adherence rates, many researchers were concerned about the possibility of drug resistance evolving. But, according to data presented by UCSF's Teri Liegler, none of the 36 men who were taking Truvada and became infected with the virus showed any signs of resistance to either tenofovir or emtricitabine, the two drugs that are combined in the Truvada pill. A separate mathematical model of the South African HIV epidemic, presented by Ume Abbas from the Cleveland Clinic Foundation in Ohio, showed that wide PrEP rollout is expected to contribute far less to drug resistance than standard antiretroviral therapy.

\section{Beyond Truvada}

Truvada is not the only possible preemptive weapon against infection. Currently, researchers are testing either Truvada or tenofovir alone in large-scale PrEP efficacy trials in 4700 Kenyan and Ugandan heterosexual couples in which one partner is HIV-positive, 2,400 injecting drug users in Thailand and 8,900 heterosexual women in sub-Saharan Africa. The results are expected over the next two years. Other PrEP candidates at earlier stages in the pipeline include Tibotec Pharmaceuticals' dapivirine, a reverse transcriptase inhibitor, and Pfizer's maraviroc, an entry inhibitor.

"As a field, we need those data," says Connie Celum of the University of Washington in Seattle, who is heading the heterosexual couple trial in East Africa. Currently, she notes, researchers only know that PrEP works in one population-gay men-so "those data are going to be critical to informing implementation."

The iPrEx trial, meanwhile, is now moving into an open-label phase where study subjects in the control arm will be offered Truvada instead of placebo. Albert Liu, a trial investigator at the San Francisco Department of Public Health, hopes that adherence rates will go up now that participants know that the drug works. The trial extension will also provide additional insights into sexual practices and drug safety, he notes.

In the initial November 2010 publication, the iPrEx investigators reported that nausea was the only serious side effect associated with the drug. However, at the meeting, UCSF metabolism researcher Kathleen Mulligan presented data showing that subjects taking Truvada had $0.5-1 \%$ lower

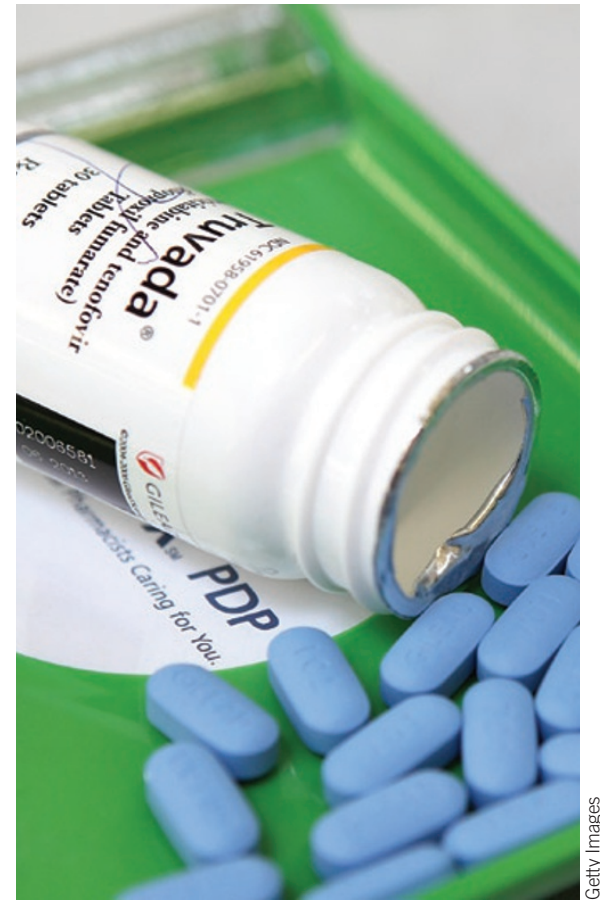

Powerful prophylaxis: Doses of Truvada.

bone mineral density compared with those receiving placebo. The two groups, however, experienced similar rates of bone fractures.

PrEP is not cheap, and determining whether it is cost effective is an important next step. Rochelle Walensky from Harvard Medical School in Boston applied the iPrEx data to South African clinical cohorts and showed that each year of prolonged life cost $\$ 3,600$ on average. "That's good value for money," she says, noting that PrEP can even be cost saving under the most favorable case scenarios.

Yet convincing doctors to prescribe PrEP could be an uphill battle. In work presented here, Kenneth Mayer, medical research director and co-chair of the Fenway Institute in Boston, one of the iPrEx study sites, surveyed 121 Massachusetts-based physicians and found that the majority of respondents preferred topical microbicides over pills to prevent HIV infection, primarily because of perceptions of fewer side effects and better ease of use.

"It's a cautionary tale," says Mayer. "Providers have some questions and concerns about PrEP administration.” But, he notes, the survey was conducted before the iPrEx results were made public. "My impression is that as people get more familiar with the data there will be a greater comfort level."

Elie Dolgin 